\title{
Multiplex Markov chains: Convection cycles and optimality
}

\author{
Dane Taylor $\circledast^{*}$ \\ Department of Mathematics, University at Buffalo, State University of New York, Buffalo, New York 14260, USA
}

(Received 23 April 2020; accepted 29 June 2020; published 29 July 2020)

\begin{abstract}
Multiplex networks are a common modeling framework for interconnected systems and multimodal data, yet we still lack fundamental insights for how multiplexity affects stochastic processes. We introduce a novel "Markov chains of Markov chains" model called multiplex Markov chains (MMCs) such that with probably $(1-\omega) \in[0,1]$ random walkers remain in the same layer and follow (layer-specific) intralayer Markov chains, whereas with probability $\omega$ they move to different layers following (node-specific) interlayer Markov chains. One main finding is the identification of multiplex convection, whereby a stationary distribution exhibits circulating flows that involve multiple layers. Convection cycles are well understood in fluids, but are insufficiently explored on networks. Our experiments reveal that one mechanism for convection is the existence of imbalances for the (intralayer) degrees of nodes in different layers. To gain further insight, we employ spectral perturbation theory to characterize the stationary distribution for the limits of small and large $\omega$, and we show that MMCs inherently exhibit optimality for intermediate $\omega$ in terms of their convergence rate and the extent of convection. As an application, we conduct an MMC-based analysis of brain-activity data, finding MMCs to differ between healthy persons and those with Alzheimer's disease. Overall, our work suggests MMCs and convection as two important new directions for network-related research.
\end{abstract}

DOI: 10.1103/PhysRevResearch.2.033164

\section{INTRODUCTION}

Convection cycles are a well-understood phenomenon in fluid dynamics [1], but they remain underexplored in the context of networks. While convection traditionally arises under external forces such as buoyancy, here we find it to emerge as a multiplexity-induced phenomenon for multiplex Markov chains (MMCs), which is a modeling framework that can be applied to study diffusion on multiplex networks. Multiplex networks [2] - a type of multilayer network [3,4] in which layers encode different types of intralayer edges, and interlayer edges couple the layers-have been used to model interconnection complex systems including transportation networks [5-7], critical infrastructures [8], and different types of relationships [9]. They also provide frameworks for data integration/fusion [10-12] and stratification [13,14].

Here, we propose a multiplex generalization of Markov chains [15], a memoryless process for stochastic transitions between discrete states that provides a theoretical foundation for diverse applications, such as queuing theory [16], population dynamics [17], and machine-learning algorithms that rely on Markov chain Monte Carlo [18], hidden Markov models [19], and/or Markov decision processes [20]. (See also Markov stability [21,22] for multiscale community

\footnotetext{
*danet@buffalo.edu

Published by the American Physical Society under the terms of the Creative Commons Attribution 4.0 International license. Further distribution of this work must maintain attribution to the author(s) and the published article's title, journal citation, and DOI.
}

detection.) Similar to Markov chains, MMCs will find diverse applications within, and beyond, the study of networks.

According to an MMC, random walkers move along (layerspecific) intralayer Markov chains with probability $\omega \in[0,1]$, and with probability $1-\omega$ they transition to new layers following (node-specific) interlayer Markov chains. MMCs can be used to study random walks on multiplex networks, and diffusion physics for multiplex networks is already a burgeoning field [23-29]. Most approaches rely on a generalization of the graph Laplacian called a supraLaplacian matrix, which can be constructed by first multiplexing the network layers' adjacency matrices into a supra-adjacency matrix, and then creating a Laplacian matrix by treating the supra-adjacency matrix as if it were a normal adjacency matrix (i.e., neglecting that intra- and interlayer edges are different). Both normalized [23] and unnormalized supraLaplacians [24-29] have been studied, and in the latter case, one can simply couple the unnormalized Laplacians of layers. Other formulations for diffusion on multiplex networks have also been proposed to study centrality and consensus [30-36]. Despite the significant advances that have been made, this field remains in its infancy [37].

By coupling "Markov-chain layers" rather than "network layers," we identify and study a novel multiplexity-induced phenomenon called multiplex convection, which-along with the convergence rate $\lambda_{2}$-is found to be optimized at intermediate $\omega$. These properties are shown to have an interesting and complicated relation to the imbalances of nodes' intralayer degrees. We analyze MMCs with spectral perturbation theory to characterize the stationary distribution when there is a separation of timescales between intra- and interlayer transitions: As $\omega \rightarrow 0$, intralayer Markov chains each approach (local) stationary solutions, and these (layer-specific) solutions are 
balanced by the interlayer Markov chains. Analogously, as $\omega \rightarrow 1$ the interlayer Markov chains individually approach (local) stationary solutions, and these (node-specific) solutions are balanced by the intralayer Markov chains.

As an application, we study frequency-multiplexed brain data through the lens of MMCs, highlighting differences between the brains of healthy persons and those with Alzheimer's disease.

This paper is organized as follows: We define MMCs in Sec. II and present our theory in Sec. III. In Sec. IV, we study the optimality of MMCs. In Sec. V, we analyze brainactivity data. We present a summary in Sec. VI. Code for the experiments is available in Ref. [38].

\section{MULTIPLEX MARKOV CHAINS}

\section{A. Model}

Consider a set of intralayer Markov chains (the "layers") with size- $N$ transition matrices $\mathbf{P}^{(i)}$ for $i \in\{1, \ldots, I\}$ and a set of (node-specific) interlayer Markov chains with size-I transition matrices $\tilde{\boldsymbol{P}}^{(n)}$ for $n \in\{1, \ldots, N\}$. Each $P_{n m}^{(i)}$ scales the transition probability from node $n$ to $m$ in layer $i$, while $\tilde{P}_{i j}^{(n)}$ scales the transition probability from layer $i$ to $j$ for node $n$. Or equivalently, these give transitions between node-layer pairs: from $(n, i)$ to $(m, i)$ in the case of $P_{n m}^{(i)}$, and from $(n, i)$ to $(n, j)$ in the case of $\tilde{P}_{i j}^{(n)}$.

A (discrete-time) multiplex Markov chain (MMC) is a stochastic process in which the states are node-layer pairs $\left(n_{p}, i_{p}\right)$, which we enumerate $p \in\{1, \ldots, N I\}$, yielding $n_{p}=$ $(p \bmod N)$ and $i_{p}=\lceil p / N\rceil(" \bmod "$ and $\lceil\cdot\rceil$ indicate the modulus and ceiling functions, respectively). Transitions between states follow a supratransition matrix

$$
\mathbb{P}(\omega)=(1-\omega) \operatorname{diag}\left(\left\{\mathbf{P}^{(i)}\right\}\right)+\omega \sum_{n} \tilde{\boldsymbol{P}}^{(n)} \otimes \mathbf{E}^{(n)} .
$$

Coupling strength $\omega \in[0,1]$ tunes the probability that random walks use interlayer vs intralayer Markov chains, $\operatorname{diag}(\cdot)$ is a block-diagonal matrix in which the argument matrices are placed along the diagonal, $\otimes$ denotes the Kronecker product, and $\mathbf{E}^{(n)}=\mathbf{e}^{(n)}\left[\mathbf{e}^{(n)}\right]^{T}$, where $\mathbf{e}_{m}^{(n)}=1$ if $n=m$ and 0 otherwise. Each $[\mathbb{P}(\omega)]_{p q}$ gives the transition probability from $\left(n_{p}, i_{p}\right)$ to $\left(n_{q}, i_{q}\right)$. Under the assumption of uniform coupling - that is, all interlayer Markov chains are identicalwe let $\tilde{\boldsymbol{P}}^{(n)}=\tilde{\boldsymbol{P}} \forall n$, and the last term in Eq. (1) simplifies to $\omega \tilde{\boldsymbol{P}} \otimes \mathbf{I}$, where $\mathbf{I}$ is a size- $N$ identity matrix.

Let $\mathbb{x}^{(t)}$ be a length-NI row vector such that $\left[\mathbb{x}^{(t)}\right]_{p}$ gives the expected fraction of random walkers at node-layer pair $\left(n_{p}, i_{p}\right)$ at time $t$. Given initial condition $\mathbb{x}^{(0)}, \mathbb{x}^{(t)}$ evolves following a linear discrete map:

$$
\mathbb{x}^{(t+1)}=\mathbb{x}^{(t)} \mathbb{P}(\omega) .
$$

If $\mathbb{P}(\omega)$ is nonnegetive, irreducible, and aperiodic, then $\mathbb{x}^{(t)} \rightarrow \mathbb{V}(\omega)$ converges to a stationary distribution, which is the left dominant eigenvector of $\mathbb{P}(\omega)$. It is convenient to define $\pi_{n_{p}}^{\left(i_{p}\right)}(\omega)=[\mathbb{v}(\omega)]_{p}$ and to drop the subscript $p$, so that $\pi_{n}^{(i)}(\omega)$ is the density of walkers at node $n$ in layer $i$.

We also define a (continuous-time) MMC with a normalized supraLaplacian

$$
\mathbb{L}(\omega)=\mathbb{I}-\mathbb{P}(\omega),
$$

where $\mathbb{I}$ is a size- $(N I)$ identity matrix. Entries $[\mathbb{L}(\omega)]_{p q}$ are rates for transitions between node-layer pairs. Letting $\mathbb{x}(t)$ denote the distribution of random walkers at time $t$, it evolves in time as $\frac{d}{d t} \mathbb{I}(t)=-\mathbb{L}(\omega)^{T} \mathbb{x}(t)$. The stationary distribution for this process is identical to that of Eq. (2). One can also define a consensus/synchronization process $[39,40]$ by $\frac{d}{d t} \mathbb{X}(t)=-\mathbb{L}(\omega) \mathbb{X}(t)$, for which the stationary distribution is the uniform distribution. For the remainder of this paper, we will focus on discrete-time MMCs due to the wealth of existing knowledge on supraLaplacians [23-29].

\section{B. Application of MMCs to multiplex networks}

Although a MMC need not be constructed from a multiplex network, MMCs do provide a new form of diffusion on multiplex networks. Let $\mathbf{P}^{(i)}=\left[\mathbf{D}^{(i)}\right]^{-1} \mathbf{A}^{(i)}$ and $\tilde{\boldsymbol{P}}^{(n)}=\left[\tilde{\boldsymbol{D}}^{(n)}\right]^{-1} \tilde{\boldsymbol{A}}^{(n)}$ denote intra-and interlayer transition matrices, respectively, of Markov chains derived from a multiplex network in which $\mathbf{A}^{(i)}$ and $\tilde{\boldsymbol{A}}^{(n)}$ are intra- and interlayer adjacency matrices, and $\mathbf{D}^{(i)}$ and $\tilde{\boldsymbol{D}}^{(n)}$ are diagonal matrices with entries $D_{n n}^{(i)}=d_{n}^{(i)}=$ $\sum_{m} A_{n m}^{(i)}$ and $\tilde{D}_{i i}=\tilde{d}_{i}^{(n)}=\sum_{j} \tilde{A}_{i j}^{(n)}$ that encode the intralayer degrees and interlayer degrees.

Before continuing, we note that there may be applications in which the transition matrices are not constructed from adjacency matrices. That is, in general an inter-or intralayer transition matrix does not necessary have take the specific functional form $P=D^{-1} A$. We therefore highlight that the study of diffusion on multiplex networks is just one of many potential applications for MMCs.

In Fig. 1(a), we visualize an MMC in which $\mathbf{P}^{(1)}$ and $\mathbf{P}^{(2)}$ are intralayer transition matrices associated with chain and star networks (both are undirected and unweighted). Selfedges are added to the first and last nodes of the chain to make it 2-regular. We enumerate the nodes $n \in\{1, \ldots, 11\}$ clockwise around the chain, starting at the center node. We implement uniform coupling with $\tilde{\boldsymbol{P}}=\left[\begin{array}{ll}0 & 1 \\ 1 & 0\end{array}\right]$. Node colors in Fig. 1(a) depict $\pi_{n}^{(i)}(\omega)$ for $\omega=0.5$.

Note that $\pi_{n}^{(i)}(\omega)$ is largest for node-layer pair $(n, i)=$ $(1,2)$ (the hub node in the star network); $\pi_{n}^{(i)}(\omega)$ is also large for node-layer pair $(1,1)$, since it is coupled to $(1,2)$ by an interlayer edge. Also, observe for layer $i=1$ that the $\pi_{n}^{(i)}(\omega)$ values monotonically decrease as one moves clockwise around the chain. That is, the stationary distributions of MMCs are influenced by global structure, which can be beneficial, for example, if one seeks to study the importances of nodes and/or layers [31-36].

As shown in Figs. 1(c) and 1(d), MMCs provide an important contrast to popular models for diffusion on multiplex networks in which one first couples the layers' adjacency matrices into a supra-adjacency $\hat{\mathbb{A}}(\omega)$, and then one subsequently defines a diffusion process that treats $\hat{\mathbb{A}}(\omega)$ as if it were a standard adjacency matrix of a single-layer network. Note that this step neglects that inter- and intralayer edges are different types of edges. We describe these models in detail in Appendix A and provide a brief summary here. This approach can give rise to a different type of supratransition matrix [32] $\hat{\mathbb{P}}(\omega)=\hat{\mathbb{D}}(\omega)^{-1} \hat{\mathbb{A}}(\omega)$, a different normalized supraLaplacian matrix $\mathbb{I}-\hat{\mathbb{P}}(\omega)$ [23], and an unnormalized supraLaplacian $\hat{\mathbb{L}}(\omega)=\hat{\mathbb{D}}(\omega)-\hat{\mathbb{A}}(\omega)$ [24-29]. [Here, $\hat{\mathbb{D}}(\omega)$ is a degree 
(a)

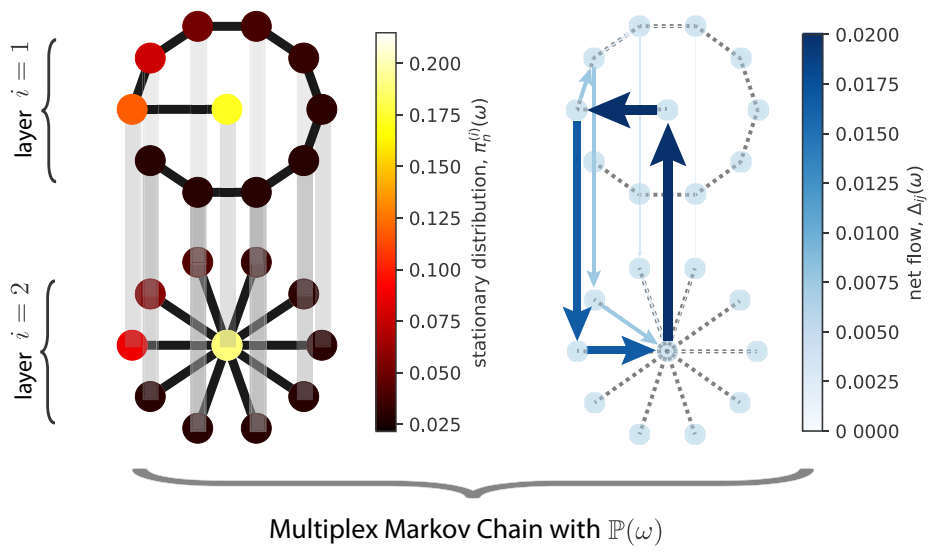

(c)

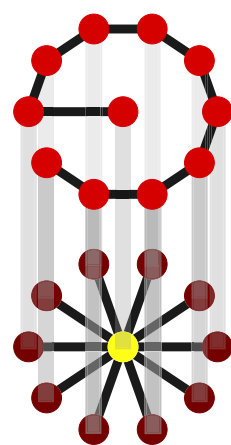

(d)

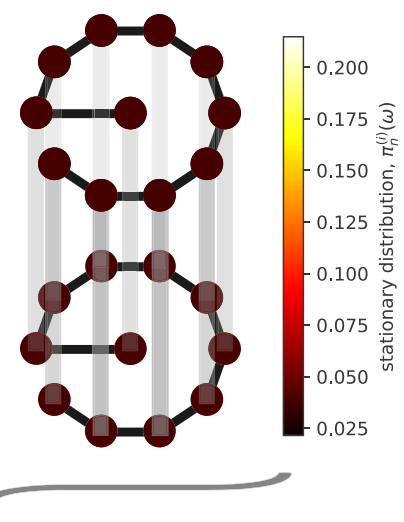

Models of Diffusion on Multiplex Networks

FIG. 1. Multiplex Markov chains (MMCs) versus diffusion models for multiplex networks. (a) Stationary distribution $\pi_{n}^{(i)}(\omega)$ for a discrete-time MMC with interlayer coupling strength $\omega=0.5$. The black and grey lines indicate edges in the intra- and interlayer Markov chains, respectively. (b) The stationary flow imbalance $\Delta_{p q}(\omega)=\pi_{n_{p}}^{\left(i_{p}\right)}(\omega)[\mathbb{P}(\omega)]_{p q}-\pi_{n_{q}}^{\left(i_{q}\right)}(\omega)[\mathbb{P}(\omega)]_{q p}$ reveals for each edge $(p, q)$ the extent to which diffusion moves in a biased direction after the system converges to its steady state. Observe the emergent multiplex convection cycle, which is a cyclical flow at stationarity that involves more than one layer. It arises due to multiplex imbalance, whereby the multiplexing of reversible Markov chains yields an irreversible MMC. See Sec. IIC for more detail. [(c) and (d)] The stationary distributions of models for diffusion on multiplex networks that first couple layers into a supra-adjacency matrix $\hat{\mathbb{A}}(\omega)$ do not exhibit convection cycles nor do they reflect global properties of the multiplex network (see Appendix A). (c) The stationary distribution of a supratransition matrix $\hat{\mathbb{P}}(\omega)=\hat{\mathbb{D}}(\omega)^{-1} \hat{\mathbb{A}}(\omega)$ $[23,32]$ is determined by the total degrees, which are encoded in the diagonal matrix $\hat{\mathbb{D}}(\omega)$. (d) The stationary distribution of an unnormalized supraLaplacian $\hat{\mathbb{L}}(\omega)=\hat{\mathbb{D}}(\omega)-\hat{\mathbb{A}}(\omega)[24-29]$ is the uniform distribution.

matrix and II is the identity matrix]. We find that these models do not give rise to emergent convection cycles, due in part to the fact that their stationary distributions do not reflect global properties of a multiplex network. For $\hat{\mathbb{P}}(\omega)$ and $\mathbb{I}-\hat{\mathbb{P}}(\omega)$, the stationary distribution is proportional to the degrees. For $\hat{\mathbb{L}}(\omega)$, it is the uniform distribution.

\section{Convection cycles in MMCs}

The $\pi_{n}^{(i)}(\omega)$ values reflect a complicated interplay between intra- and interlayer Markov chains, which we further study through the stationary flows across edges

$$
\mathbb{F}_{p q}(\omega)=\pi_{n_{p}}^{\left(i_{p}\right)}(\omega)[\mathbb{P}(\omega)]_{p q} .
$$

We further define $\mathbb{F}(\omega)=\overline{\mathbb{F}}(\omega)+\Delta(\omega) / 2$ to separate the matrix into its symmetric part, $\overline{\mathbb{F}}(\omega)=\left[\mathbb{F}(\omega)+\mathbb{F}(\omega)^{T}\right] / 2$, and skew-symmetric part, $\Delta(\omega) / 2$. Each entry

$$
\Delta_{p q}(\omega)=\mathbb{F}_{p q}(\omega)-\mathbb{F}_{q p}(\omega)
$$

indicates the stationary flow imbalance across each edge. $\Delta_{p q}(\omega)>0$ implies that there is a greater flow from $\left(n_{p}, i_{p}\right)$ and $\left(n_{q}, i_{q}\right)$, whereas $\Delta_{p q}(\omega)<0$ implies the opposite. To shorten our notation, in the rest of the paper we will drop the argument $\omega$ in $\mathbb{F}(\omega)$ and $\Delta(\omega)$.

We quantify the total flow imbalance $\|\Delta\|_{F}$ using the Frobenius norm, and a Markov chain is reversible iff $\|\Delta\|_{F}=$ 0 (i.e., $\Delta_{p q}=0 \forall p, q$ ). We define multiplex imbalance as the phenomenon whereby the multiplex coupling of reversible Markov chains yields an irreversible MMC. In that case, $\|\Delta\|_{F}$ quantifies the total multiplex imbalance.

In Fig. 1(b), we visualize $\Delta_{p q}$ for the MMC from Fig. 1(a). Observe that these values exhibit a circulating flow imbalance involving more than one layer, which we call multiplex convection. Because $\mathbf{P}^{(1)}, \mathbf{P}^{(2)}$ and $\tilde{\boldsymbol{P}}$ are all transition matrices of reversible Markov chains, the irreversibility of the MMC is an emergent multiplexity-induced property.

Note that $\Delta_{p q}$ is largest from node-layer pair $(1,2)$ to $(1,1)$, and this edge is also associated with the largest imbalance of intralayer degrees: $d_{1}^{(2)}=10, d_{1}^{(1)}=2$, and $d_{1}^{(2)}-d_{1}^{(1)}=8$ (recall the chain layer has self-edges to make it 2-regular). This reveals an important mechanism that contributes to the emergence of multiplex imbalance and convection: $\Delta_{p q}$ is often large for an interlayer edge associated with a node $n$ such that its intralayer degrees $d_{n}^{\left(i_{p}\right)}$ and $d_{n}^{\left(i_{q}\right)}$ are imbalanced (where $d_{n}^{(i)}=\sum_{m} A_{n m}^{(i)}$ ).

\section{TIMESCALE SEPARATION ANALYSIS}

We analyze $\pi_{n}^{(i)}(\omega)$ for two limits: as $\omega \rightarrow 0$ : random walkers rarely move between layers, yielding a type of layer decoupling. As $\omega \rightarrow 1$ : random walkers rarely remain in the same layer, yielding a type of layer aggregation. To this end, we develop spectral perturbation theory in Appendix B. Here, we will summarize these mathematical results.

Given intra- and interlayer Markov chains with transition matrices $\mathbf{P}^{(i)}$ and $\tilde{\boldsymbol{P}}^{(n)}$, respectively, let $\mathbf{v}^{(i)}$ and $\tilde{\boldsymbol{v}}^{(n)}$ denote their stationary distributions. Note that they are left eigenvectors associated with an eigenvalue equal to 1 . Let $\tilde{\boldsymbol{e}}^{(i)}$ be a length- $I$ unit vector (i.e., $\tilde{e}_{j}^{(i)}=1$ if $j=i$ and 0 otherwise). Setting $\omega=0$ in Eq. (1) yields $\mathbb{P}(0)=\operatorname{diag}\left(\left\{\mathbf{P}^{(i)}\right\}\right)$, for which $\lambda_{1}=1$ is an eigenvalue. With Theorem B.1 in Appendix B, we show that $\lambda_{1}$ has an $I$-dimensional left eigenspace spanned by vectors

$$
\mathbb{V}^{(i)}=\tilde{\boldsymbol{e}}^{(i)} \otimes \mathbf{v}^{(i)}
$$

However, for any positive $\omega$, Perron-Frobenius theory [41] ensures that the eigenvalue $\lambda_{1}=1$ of $\mathbb{P}(\omega)$ has a 
one-dimensional eigenspace spanned by a unique left dominant eigenvector $\mathbb{v}(\omega)$. Moreover, $\mathbb{v}(\omega)$ must converge within the subspace $\operatorname{span}\left(\mathbb{V}^{(i)}\right)$, implying there exist constants $\tilde{\alpha}_{i}$ such that $\lim _{\omega \rightarrow 0} \mathbb{V}(\omega)=\sum_{i} \tilde{\alpha}_{i} \mathbb{V}^{(i)}$. We denote $\tilde{\boldsymbol{\alpha}}=\left[\tilde{\alpha}_{1}, \ldots, \tilde{\alpha}_{I}\right]$.

With Theorem B.3 in Appendix B, we show that $\tilde{\boldsymbol{\alpha}}$ is the dominant left eigenvector of an "effective" interlayer transition matrix $\tilde{X}$ with entries $\tilde{X}_{i j}=\sum_{n} v_{n}^{(i)} \tilde{P}_{i j}^{(n)}$. Using the notation $[\mathbb{v}(\omega)]_{p}=\pi_{n_{p}}^{\left(i_{p}\right)}(\omega)$, we have

$$
\lim _{\omega \rightarrow 0} \pi_{n}^{(i)}(\omega)=\tilde{\alpha}_{i} v_{n}^{(i)}
$$

That is, each intralayer Markov chain $i$ obtains its stationary distribution $\mathbf{v}^{(i)}$, and these local solutions are balanced by the stationary distribution of an "effective" interlayer Markov chain that depends on all inter- and intralayer Markov chains. In the case of uniform coupling, $\tilde{\boldsymbol{P}}^{(n)}=\tilde{\boldsymbol{P}}, \tilde{\boldsymbol{X}}=\tilde{\boldsymbol{P}}$, and $\tilde{\boldsymbol{\alpha}}=\tilde{\boldsymbol{v}}$ (the left dominant eigenvector of $\tilde{\boldsymbol{P}}$ ).

We analyze the limit $\omega \rightarrow 1$ in a similar way, except we first implement a change of basis via the (unitary) stridepermutation matrix $\mathbb{U}$ that reorders the node-layer pairs as layer-node pairs: $[\mathbb{U}]_{p q}=1$ if $q=\lceil p / N\rceil+T[(p-1) \bmod$ $N]$ and $[\mathbb{U}]_{p q}=0$ otherwise. Matrix $\mathbb{Q}(\omega)=\mathbb{U} \mathbb{P}(\omega) \mathbb{U}^{T}$ is a supratransition matrix for the same MMC as $\mathbb{P}(\omega)$; the only difference is that the rows and columns have been permuted. We obtain

$$
\mathbb{Q}(\omega)=(1-\omega) \sum_{i} \mathbf{P}^{(i)} \otimes \tilde{\boldsymbol{E}}^{(i)}+\omega \operatorname{diag}\left(\left\{\tilde{\boldsymbol{P}}^{(n)}\right\}\right),
$$

where $\tilde{\boldsymbol{E}}^{(i)}=\tilde{\boldsymbol{e}}^{(i)}\left[\tilde{\boldsymbol{e}}^{(i)}\right]^{T}$ and $\tilde{\boldsymbol{e}}^{(i)}$ is a length-I unit vector. Observe that the form of $\mathbb{Q}(\omega)$ qualitatively matches that of Eq. (1); only the inter- and intralayer transition matrices have been swapped. Thus, one can equally interpret an MMC as intralayer Markov chains coupled by intralayer ones, or as interlayer Markov chains coupled by intralayer ones. These are formally the same. We can thus make use of our earlier results to obtain

$$
\lim _{\omega \rightarrow 1} \pi_{n}^{(i)}(\omega)=\alpha_{n} \tilde{v}_{i}^{(n)},
$$

where $\boldsymbol{\alpha}=\left[\alpha_{1}, \ldots, \alpha_{N}\right]$ is the dominant left eigenvector of a transition matrix $\mathbf{X}$ with entries $X_{n m}=\sum_{i} \tilde{v}_{i}^{(n)} P_{n m}^{(i)}$ for an "effective" intralayer Markov chain.

In Fig. 2, we validate the accuracy of Eqs. (7) and (9) for the MMC shown in Fig. 1. The observed $\pi_{n}^{(i)}(\omega)$ values for small and large $\omega$ were computed with $\omega=10^{-3}$ and $\omega=1-10^{-3}$.

Equations (7) and (9) have the important consequence of implying that $\lambda_{2}(\omega)$ - the second-largest-in-magnitude eigenvalue of $\mathbb{P}(\omega)$-is optimized at some intermediate value of $\omega$. Because $\mathbb{x}_{p}^{(t)} \rightarrow \pi_{n_{p}}^{\left(i_{p}\right)}(\omega)$ with $t \rightarrow \infty$ as $\mathcal{O}\left(\lambda_{2}^{t}(\omega)\right)$, $\lambda_{2}(\omega) \in(0,1)$ is called the convergence rate. Importantly, because $\mathbb{P}(w)$ has $I$-dimensional and $N$-dimensional dominant eigenspaces when $\omega=0$ and $\omega=1$, respectively, it follows that $\lambda_{2}(\omega) \rightarrow 1$ in either limit. Finally, Rolle's theorem [42] implies there is a minimum since $d \lambda_{2}(\omega) / d \omega<1$ at $\omega=$ 0 and $d \lambda_{2}(\omega) / d \omega>1$ at $\omega=1$. We study this optimality for MMCs, as well as another type of optimality, in the next section.
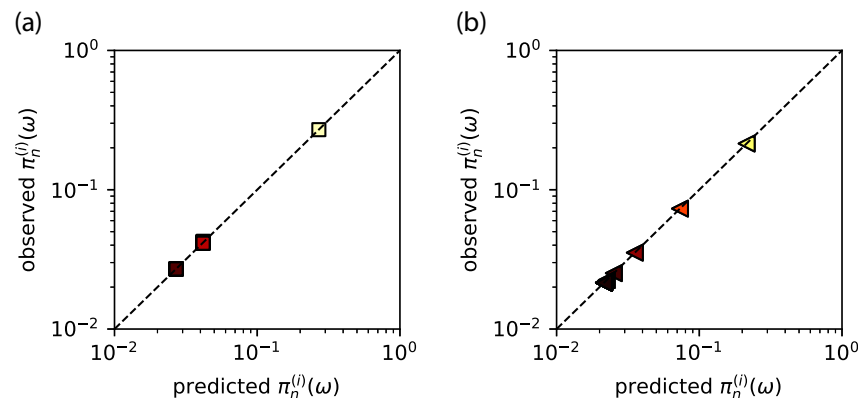

FIG. 2. Validation of theory for timescale separations. We compare observed and predicted values of $\pi_{n}^{(i)}(\omega)$ for the MMC from Fig. 1 for two limiting cases: (a) Eq. (7) for $\omega \rightarrow 0$ and (b) Eq. (9) for $\omega \rightarrow 1$. The symbols' colors correspond to the same color scale as that shown in Fig. 1(a). Note that 22 symbols are plotted in each panel, but because they overlap, we perceive just a few.

\section{OPTIMALITY OF MMCS FOR INTERMEDIATE $\omega$}

Next, we show that total multiplex imbalance $\|\Delta\|_{F}$ is maximized at some value $\omega_{\Delta}^{*}=\operatorname{argmax}_{\omega}\|\Delta\|_{F} \in(0,1)$. We use the same interlayer Markov chains as in Fig. 1, but now allow the interlayer Markov chains to be different for every node: $\tilde{\boldsymbol{P}}^{(n)}=\left[\begin{array}{cc}\left(1-a_{n}\right) & a_{n} \\ a_{n} & \left(1-a_{n}\right)\end{array}\right]$, where $a_{n} \in[a, 1]$ tunes the probability of switching layers at each node $n$. We consider four strategies for choosing $a_{n}$ :

(I) identical $a_{n}$ : we define $a_{n}=a$ for each $n$;

(II) increasing $\boldsymbol{a}_{\boldsymbol{n}}$ : we define $a_{n}=a+(n-1) \delta a$ for $n \in$ $\{1, \ldots, N\}$ with $\delta a=(1-a) /(N-1)$;

(III) decreasing $\boldsymbol{a}_{\boldsymbol{n}}$ : we let $a_{n}=1-(n-1) \delta a$; and

(IV) random $a_{n}$ : we sample $a_{n}$ uniformly at random from $[a, 1]$.

In Fig. 3(a), we plot $\|\Delta\|_{F}$ versus $\omega$; each panel depicts a different strategy. First, because $a \rightarrow 1$ recovers the interlayer transition matrix used in Fig. 1 for all strategies, the (blue solid) curves for $a=0.99$ are nearly identical in all top panels. As $a$ decreases, the different strategies yield different $\|\Delta\|_{F}$ curves: [(I) and (II)] the curves for identical and increasing $a_{n}$ flatten as $a$ decreases and the location of the optimum shifts toward larger $\omega$; (III) the $\|\Delta\|_{F}$ curves for decreasing $a_{n}$ are insensitive to $a$; and (IV) the curves for random $a_{n}$ seem to change randomly, but generally decrease. These responses can be understood by noting that $a_{1}$ strongly varies with $a$ for the first two strategies, it remains unchanged for the decreasing- $a_{n}$ strategy, and it is random for the last strategy, although its expectation decreases. Parameter $a_{1}$ determines the optimality of $\|\Delta\|_{F}$ in this case, because the net flow $\Delta_{p q}$ is largest from node-layer pair $(1,2)$ to $(1,1)$ [see Fig. $1(\mathrm{~b})$ ], and $a_{1}$ tunes the probability of walkers make this transition.

In Fig. 3(b), we show $\lambda_{2}(\omega)$ versus $\omega$ for the same MMC as in Fig. 3(a). Interestingly, the locations $\omega_{\Delta}^{*}$ and $\omega_{\lambda_{2}}^{*}$ of optima for $\|\Delta\|_{F}$ and $\lambda_{2}(\omega)$ appear to be strongly correlated for some strategies. We now explore this further by repeating this experiment with many values of $a \in(0,1)$.

In Figs. 4(a) and 4(b), we plot $\omega_{\Delta}^{*}$ and $\omega_{\lambda_{2}}^{*}$, respectively, as a function of $a$. In each panel, we show results for the four strategies for creating interlayer Markov chains. Observe that in the limit $a \rightarrow 1$, all of the optimums occur at approximately the same coupling strength $\omega_{\lambda_{2}}^{*} \approx \omega_{\Delta}^{*} \approx 0.4$, which 

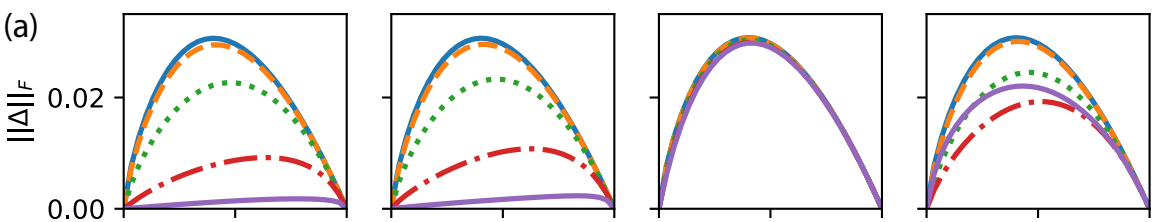

(b)
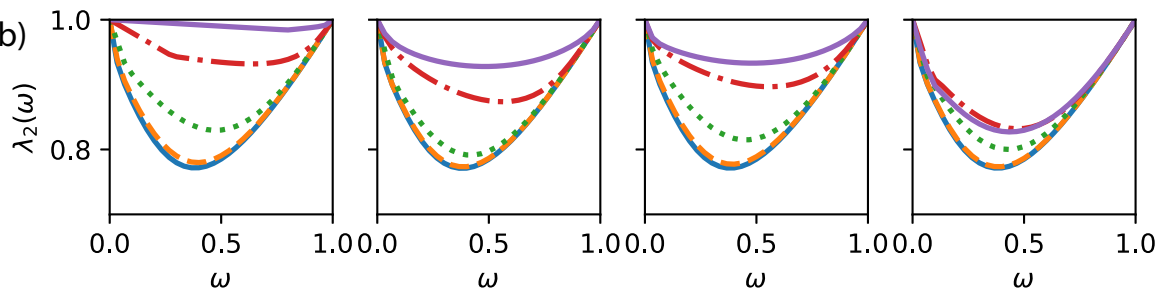

$-a=0.99$ $\omega$ $\omega$ $\omega$

$a=0.1$

$a=0.01$
FIG. 3. Optimal imbalance and convergence rate. (a) Total multiplex imbalance $\|\Delta\|_{F}$ and (b) convergence rate $\lambda_{2}(\omega)$ vs $\omega$ for the same intralayer Markov chains as in Fig. 1, but with node-specific interlayer Markov chains in which $a_{n}$ tunes the probability of switching layers at node $n$. We choose $a_{n} \in[a, 1]$ via four strategies: (left) identical $a_{n}: a_{n}=a \forall n$; (center-left) increasing $a_{n}$ : evenly spaced and monotonically increasing with $n$; (center-right) decreasing $a_{n}$ : evenly spaced and monotonically decreasing with $n$; and (right) random $a_{n}$ : sampled uniformly at random. We define $\omega_{\Delta}^{*}=$ $\operatorname{argmax}_{\omega}\|\Delta\|_{F}$ and $\omega_{\lambda_{2}}^{*}=\operatorname{argmax}_{\omega} \lambda_{2}(\omega)$. is expected since all four strategies yield uniform coupling: $\tilde{\boldsymbol{P}}^{(n)} \rightarrow \tilde{\boldsymbol{P}}=\left[\begin{array}{ll}0 & 1 \\ 1 & 0\end{array}\right]$. As one decreases $a$, the optimums shift to larger values of $\omega$ for all strategies except for the strategy with decreasing $a_{n}$. This response is largest for the strategies with identical $a_{n}$ and increasing $a_{n}$, and it is less clear for the strategy with random $a_{n}$ (in which case, the dependence of $\omega_{\Delta}^{*}$ on $a$ is more random).

In Fig. 4(c), we compare $\omega_{\lambda_{2}}^{*}$ and $\omega_{\Delta}^{*}$ across these values of $a$. Note for the strategy of identical $a_{n}$, that the two the optimums occur at nearly the same value of $\omega$ for any $a \in$ $(0,1)$ - that is, the blue squares lie along the diagonal. The dependence of $\omega_{\lambda_{2}}^{*}$ and $\omega_{\Delta}^{*}$ on $a$ is also strongly correlated for the strategy of increasing $a_{n}$; however $\omega_{\Delta}^{*}$ is always slightly larger than $\omega_{\lambda_{2}}^{*}$ and the relationship appears to be nonlinear for small $a$. The relation between $\omega_{\lambda_{2}}^{*}$ and $\omega_{\Delta}^{*}$ appears to be random for the random strategy. Interestingly, for the strategy of decreasing $a_{n}, \omega_{\lambda_{2}}^{*}$ clearly increases with decreasing $a$, however $\omega_{\Delta}^{*}$ appears to not depend on $a$.

We give the following interpretation to provide intuitive insight into these results. Recall from Fig. 1(b) that the imbalance $\Delta_{p q}$ is largest for the edge connecting node 1 in layer 2 to node 1 in layer 1 . That imbalance requires a net flow from node-layer pair $(1,2)$ to $(1,1)$. Since $a_{1}$ tunes the transition rate between layers at node $n=1$, this net flow will monotonically increase with $a_{1}$. Therefore we expect the multiplex imbalance to be most sensitive to $a$ when $a_{1}$ changes with $a$. Parameter $a_{1}$ is most sensitive to $a$ for the strategies of identical $a_{n}$ and increasing $a_{n}$ (i.e., $a_{1}=a$ in these cases), it randomly depends on $a$ for the strategy of random $a_{n}$ (although it increases in expectation since $\mathbb{E}\left[a_{n}\right]=$ $(1+a) / 2)$, and it does not vary with $a$ for the strategy of decreasing $a_{n}$ (i.e., $a_{1}=1$ ). Therefore our observed sensitivity with $a$ for the different strategies is is exactly as one would expect based on our understanding for how degree imbalances affect flow imbalances.

\section{APPLICATION TO BRAIN-ACTIVITY DATA}

We now study a MMC representation of a functional brain network [13] with $N=148$ nodes (brain regions) and $I=7$ layers. The data include pairwise coherences of magnetoencephalography (MEG) signals at different frequency ranges, $\{[1,4),[4,8),[8,10.5),[10.5,13),[13,20),[20,30),[30,45)\}$ (measured in $\mathrm{Hz}$ ), and we interpret the matrices as intralayer adjacency matrices. We construct intralayer transition matrices for them as described in Sec. II B.

We uniformly couple the layers with an interlayer Markov chain with transition matrix

$$
\tilde{P}_{i j}=\left\{\begin{aligned}
1, & |i-j|=1 \text { and } i \in\{1,7\} \\
1 / 2, & |i-j|=1 \text { and } i \in\{2, \ldots, 6\} . \\
0, & |i-j| \neq 1
\end{aligned}\right.
$$

In Appendix C, we study node-specific transition matrices $\tilde{\boldsymbol{P}}^{(n)}$ that are similar to those described in Sec. IV.
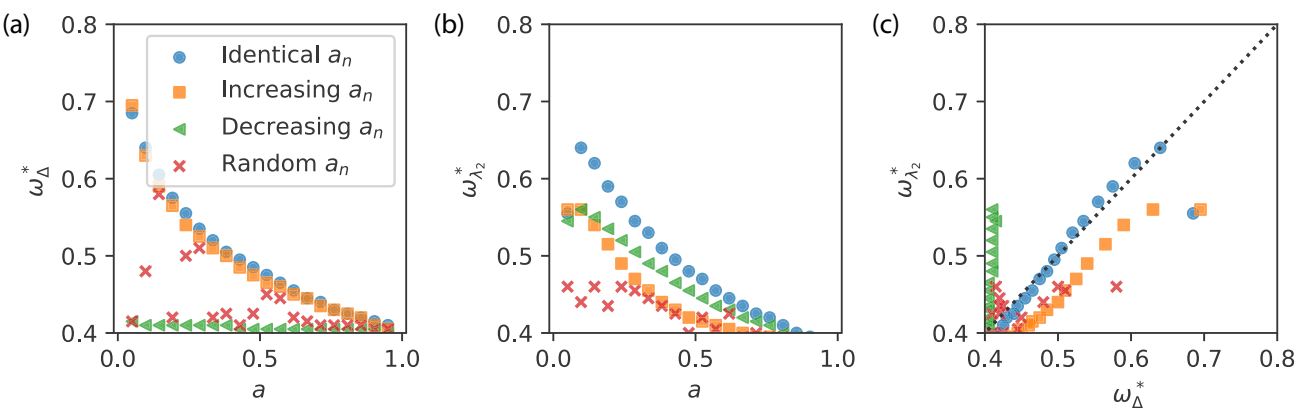

FIG. 4. Relationship between the optimum for multiplex imbalance and the optimum for convergence rate. We show results for hetergeneous interlayer Markov chains in which the probably to move to a different layer at each node $n$ is tuned by $a_{n}$, and we study four strategies for selecting $a_{n}$, each of which depends on a $a$. Panels (a) and (b) depict $\omega_{\Delta}^{*}=\operatorname{argmax}_{\omega}\|\Delta\|_{F}$ and $\omega_{\lambda_{2}}^{*}=\operatorname{argmax}_{\omega} \lambda_{2}(\omega)$, respectively, as a function of parameter $a$. Panel (c) depicts a direct comparison of $\omega_{\Delta}^{*}$ and $\omega_{\lambda_{2}}^{*}$. 

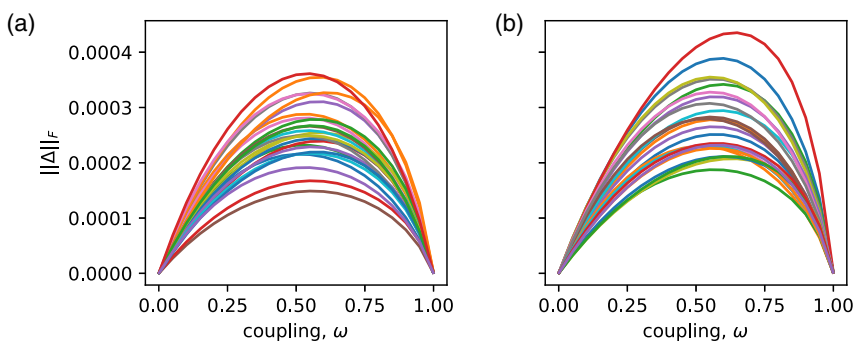

FIG. 5. Optimality is different for persons with and without Alzheimers. We plot $\|\Delta\|_{F}$ vs $\omega$ for 25 persons (a) healthy persons and (b) persons with Alzheimer's disease. Observe that the $\|\Delta\|_{F}$ values are slightly larger for persons with the disease, and the value of $\omega$ at which the optimum occurs, $\omega_{\Delta}^{*}$, shifts slightly to the right.

We first conducted a population-level study of the optimality of MMCs for the 50 persons in the dataset [13]: 25 healthy persons and 25 persons with Alzheimer's disease. In Fig. 5, we plot $\|\Delta\|_{F}$ versus $\omega$ for (a) healthy persons and (b) those with Alzheimer's disease. Observe that the $\|\Delta\|_{F}$ values are slightly larger for persons with the disease, and the value of $\omega$ at which the optimum occurs, $\omega_{\Delta}^{*}$, shifts slightly to the right. Specifically, when $\omega=0.5,\|\Delta\|_{F}$ is $6.6 \%$ larger for persons with Alzheimers (0.000276 versus 0.000259). The average of $\omega_{\Delta}^{*}=\operatorname{argmax}_{\omega}\|\Delta\|_{F}$ was also found to be $2.1 \%$ larger for persons with Alzheimers ( 0.570 versus 0.558$)$.

Next, we study the extent to which degree imbalance is a mechanism that helps drive the different optimality of MMCs for healthy and diseased brains. Recall our discussion in the last paragraph of Sec. IIC for how degree imbalance helped create the convection cycle for the MMC that was shown in Fig. 1(b).

Now, we will show that a similar phenomenon occurs for the MMC representations of the brain data. That is, $\Delta_{p q}$ is often large for an interlayer edge associated with a node $n$ such that its intralayer degrees $d_{n}^{\left(i_{p}\right)}$ and $d_{n}^{\left(i_{q}\right)}$ are imbalanced.

In Fig. 6, we plot $\Delta_{p q}$ versus $\left(d_{n_{p}}^{\left(i_{p}\right)}-d_{n_{q}}^{\left(j_{q}\right)}\right)$ separately for (a) intralayer edges (i.e., $i_{p}=i_{q}$ ) and (b) interlayer edges (i.e., $n_{p}=n_{q}$ ). Different columns show results for different $\omega$. First, observe that $\Delta_{p q}$ are approximately $50 \times$ larger for interlayer edges than for intralayer edges [i.e., compare the $y$ axis of (b) to that of (a)]. Also, note that $\Delta_{p q}$ obtain their largest magnitudes near $\omega=0.5$ (center column), which is consistent with our finding that $\omega_{\Delta}^{*} \approx 0.5$ for this MMC. In Appendix $\mathrm{C}$, we present extended results by studying the separate optimality of each $\Delta_{p q}$ versus $\omega$.

The strong correlation between $\Delta_{p q}$ and $\left(d_{n}^{\left(i_{p}\right)}-d_{n}^{\left(j_{q}\right)}\right)$ supports our hypothesis that the largest $\Delta_{p q}$ occur for interlayer edges associated with the largest intralayer-degree imbalance, $\left(d_{n}^{\left(i_{p}\right)}-d_{n}^{\left(j_{q}\right)}\right)$. We also find that this correlation differs between healthy and diseased brains. In Fig. 7, we plot the Pearson correlation coefficient between $\Delta_{p q}$ and $\left(d_{n_{p}}^{\left(i_{p}\right)}-\right.$ $d_{n_{q}}^{\left(j_{q}\right)}$ ) versus $\omega$ for (a) healthy persons and (b) persons with Alzheimer's disease. Different curves correspond to different people. The thick colored curves indicate the subpopulations' mean values, and they are repeatedly shown in Fig. 7(c) to highlight the difference between persons with/without the disease. We separately computed these correlation coefficients for intralayer and interlayer edges, which we show in the upper and lower rows, respectively.

Given our observation that imbalanced intralayer degrees contribute to multiplex imbalance and convection, and that both are increased for persons with Alzheimer's disease, our findings are consistent with previous work that found persons with Alzheimer's disease to have a loss of brain interfrequency hubs [13].

\section{CONCLUSION}

Our work is motivated by interdisciplinary applications that use discrete Markov chains [16-20] and by the observation that existing multiplex diffusion models [24-31] are limited in their behavior (see Fig. 1). Here, we introduced a multiplex generalization of Markov chains that revealed novel phenomena: multiplex convection and imbalance. Convection cycles are a central topic in fluid mechanics, but they remain underexplored on networks. We identified degree imbalances as one mechanism that contributes to convection, we showed that both the extent of convection and the convergence rate are optimized at intermediate coupling $\omega$. Finally, we developed an MMC-based study of frequency-multiplexed brain-activity data, finding that that the MMCs for persons with Alzheimer's disease differ from those of healthy persons. Our work highlights MMCs and convection as two important new directions for network-science research. (a)

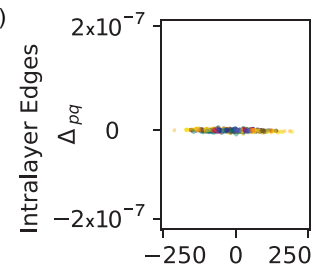

(b)

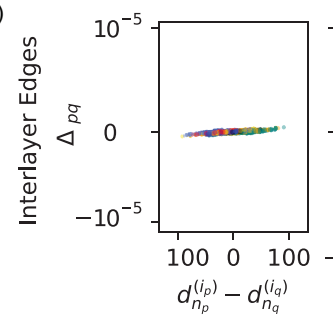

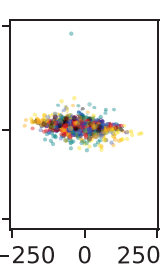
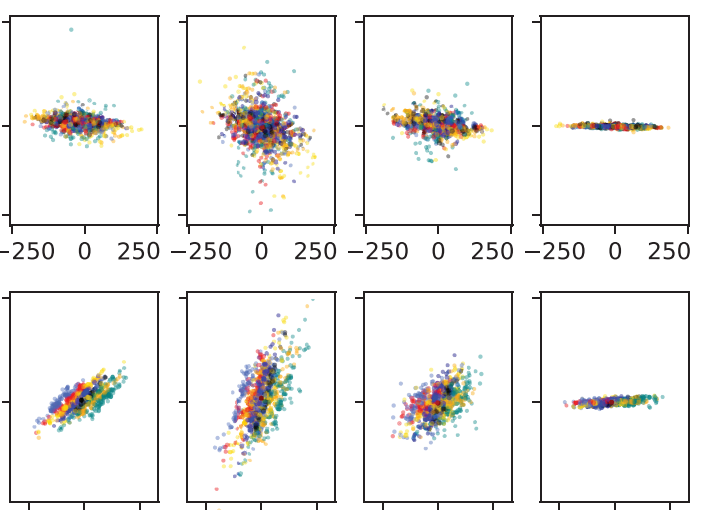

$\begin{array}{ccc}100 & 0 & 100 \\ d^{\left(i_{p}\right)}-d^{\left(i_{q}\right)}\end{array}$
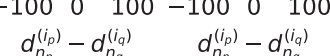

$-1000 \quad 100$ $d_{n_{p}}^{\left(i_{p}\right)}-d_{n_{q}}^{\left(i_{q}\right)}$
FIG. 6. Degree imbalance contributes to multiplex imbalance and convection for an MMC representation of brain-activity data. We separately plot $\Delta_{p q}$ versus $\left(d_{n_{p}}^{\left(i_{p}\right)}-d_{n_{q}}^{\left(i_{q}\right)}\right)$ for (a) intralayer edges and (b) interlayer edges for a MMC representation of an empirical brain network with $N=148$ nodes (brain regions) and $I=7$ layers (correlated MEG signals at different frequency ranges). From left to right, the different columns show results for different coupling strength: $\omega \in\{0.01,0.1,0.5,0.9,0.99\}$. Observe that $\Delta_{p q}$ and $\left(d_{n_{p}}^{\left(i_{p}\right)}-d_{n_{q}}^{\left(i_{q}\right)}\right)$ are positively (negatively) correlated for interlayer (intralayer) edges, and $\Delta_{p q}$ have their largest magnitudes for $\omega=0.5$ (center column). 


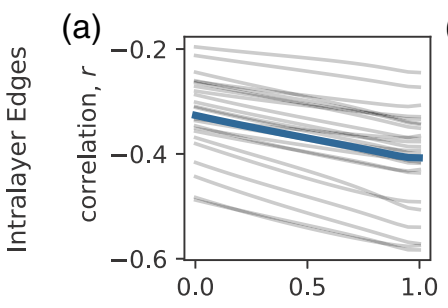

(b)
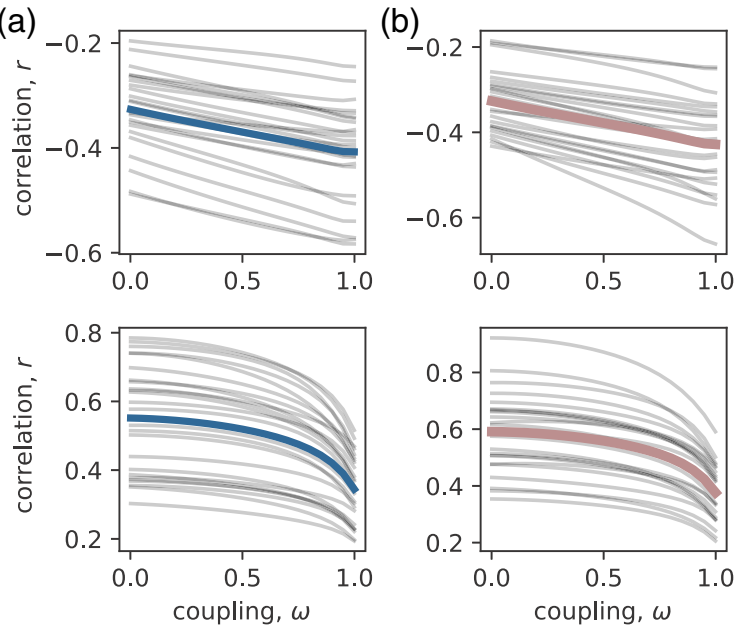

(c)

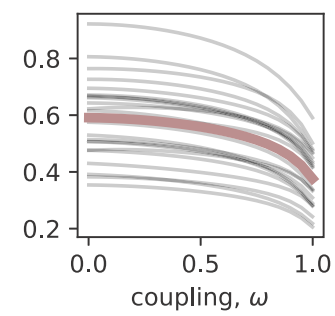

\section{ACKNOWLEDGMENTS}

We thank Per Sebastian Skardal, Naoki Masuda, and Sarah Muldoon, as well as the referees, for their helpful feedback. This work was supported in part by the Simons Foundation (Grant No. 578333).

\section{APPENDIX A: COMPARISON TO DIFFUSIONS ON MULTIPLEX NETWORKS}

The diffusion models that most closely resemble MMCs are the ones that first define a supra-adjacency matrix

$$
\widehat{\mathbb{A}}(\omega)=(1-\omega) \operatorname{diag}\left(\left\{\mathbf{A}^{(i)}\right\}\right)+\omega \sum_{n} \tilde{\boldsymbol{A}}^{(n)} \otimes \mathbf{E}^{(n)}
$$

that couples intralayer adjacency matrices $\left\{\mathbf{A}^{(i)}\right\}$ with interlayer adjacency matrices $\left\{\tilde{\boldsymbol{A}}^{(n)}\right\}$. Then one defines a transition matrix by neglecting that the inter- and intralayer edges are different,

$$
\hat{\mathbb{P}}(\omega)=\hat{\mathbb{D}}(\omega)^{-1} \hat{\mathbb{A}}(\omega),
$$

where $\hat{\mathbb{D}}(\omega)$ is a diagonal matrix in which the diagonal entries encode the nodes' total degrees,

$$
[\hat{\mathbb{D}}(\omega)]_{p p}=\sum_{q}[\hat{\mathbb{A}}(\omega)]_{p q}=(1-\omega) d_{n_{p}}^{\left(i_{p}\right)}+\omega \tilde{d}_{i_{p}}^{\left(n_{p}\right)} .
$$

Equation (A2) is actually slightly different from the one defined in Refs. [23,32], but theirs can be recovered by dividing $\hat{A}(\omega)$ by $(1-\omega)$, so that their coupling strength is equivalent to $\omega /(1-\omega) \in[0, \infty)$. We use the definition of Eq. (A2) since it allows us to study the same range of $\omega$ as for MMCs, $\omega \in[0,1]$. Also, it is worth noting that [23] studied a continuous-time random walk with the goal of extending Markov stability [21], whereas here we study a discrete-time random walk similar to [32]. We also note that these previous works focused on when the layers were uniformly coupled, $\tilde{\boldsymbol{A}}^{(n)}=\tilde{\boldsymbol{A}} \forall n$.

Because $\hat{\mathbb{A}}(\omega)$ is an adjacency matrix for an undirected network, $\hat{\mathbb{P}}(\omega)$ has a stationary distribution with entries that are proportional to the degrees [15]—or in this case, the node-layer pairs:

$$
\hat{\pi}_{n}^{(i)}(\omega) \propto(1-\omega) d_{n}^{(i)}+\omega \tilde{d}_{i}^{(n)} .
$$
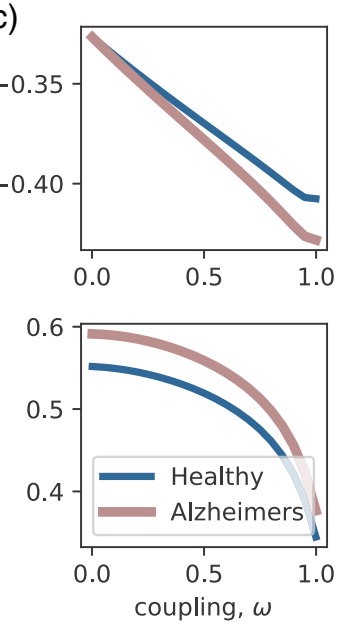

FIG. 7. Correlation between multiplex imbalance and intralayer-degree imbalance. We plot the Pearson correlation coefficient $r$ between $\Delta_{p q}$ and $d_{n_{p}}^{\left(i_{p}\right)}-d_{n_{q}}^{\left(j_{q}\right)}$ vs $\omega$ for (a) healthy persons and (b) persons with Alzheimer's disease. Different gray curves correspond to different people, and the thick colored curves indicate the mean curves. (c) A comparison of the mean curves for the two subpopulations. All correlation coefficients are separately calculated for the (top row) intralayer edges and (bottom row) interlayer edges.

Recall that $d_{n}^{(i)}=\sum_{m} A_{n m}^{(i)}$ and $\tilde{d}_{i}^{(n)}=\sum_{j} \tilde{A}_{i j}^{(n)}$ are the intralayer and interlayer degrees, respectively. In other words, the $\hat{\pi}_{n}^{(i)}(\omega)$ values are not informative of a multiplex network's global (i.e., nonlocal) properties.

\section{APPENDIX B: PERTURBATION THEORY FOR TIMESCALE SEPARATION}

We first study the dominant eigenvector of a supratransition matrix $\mathbb{P}(\omega)$ in the limit $\omega \rightarrow 0^{+}$, which corresponds to when transitions rarely occur using an interlayer Markov chain.

Let $\mu_{1}^{(i)}, \mathbf{v}^{(i)}$, and $\mathbf{u}^{(i)}$ denote the largest positive eigenvalue and corresponding left and right eigenvectors of each transition matrix $\mathbf{P}^{(i)}$ of the intralayer Markov chains, where $i \in\{1, \ldots, I\}$ is a layer index. Furthermore, let $\tilde{\mu}_{1}^{(n)}, \tilde{\boldsymbol{v}}^{(n)}$, and $\tilde{\boldsymbol{u}}^{(n)}$ denote the same mathematical elements for each transition matrix $\tilde{\boldsymbol{P}}^{(n)}$ of the interlayer Markov chains, where $n \in\{1, \ldots, N\}$ is a node indicx. Note that $\mu_{1}^{(i)}=\tilde{\mu}_{1}^{(n)}=$ 1 for any $i$ and $n$, since the transition matrices are row stochastic. Also, their corresponding right eigenvectors $\mathbf{u}^{(i)}=$ $[1, \ldots, 1]^{T}$ and $\tilde{\boldsymbol{u}}^{(n)}=[1, \ldots, 1]^{T}$ are vectors in which all the entries are ones. It is advantageous to let the left eigenvectors represent probability distributions, and so we normalize them in 1-norm. We do not normalize the right eigenvectors (i.e., $\sum_{n} u_{n}^{(i)}=N$ and $\left.\sum_{i} \tilde{u}_{i}^{(n)}=I\right)$ so that $\left[\mathbf{v}^{(i)}\right]^{T} \mathbf{u}^{(i)}=\sum_{n} v_{n}^{(i)}=$ 1 and $\left[\tilde{\boldsymbol{v}}^{(n)}\right]^{T} \tilde{\boldsymbol{u}}^{(n)}=\sum_{i} \tilde{\boldsymbol{v}}_{i}^{(n)}=1$ for any $i$ and $n$. Provided that the transition matrices $\mathbf{P}^{(i)}$ and $\tilde{\boldsymbol{P}}^{(n)}$ are nonnegative and irreducible, Perron-Frobenius theory for nonnegative matrices [41] guarantees that the left eigenvectors $\mathbf{v}^{(i)}$ and $\widetilde{\boldsymbol{v}}^{(n)}$ are unique and contain positive entries.

Turning our attention to the spectra of $\mathbb{P}(\omega)$, we denote its largest positive eigenvalue by $\lambda_{1}(\omega)$ and its left and right eigenvectors by $\mathbb{v}(\omega)$ and $u(\omega)$, respectively. We can write the dominant eigenvector equations as

$$
\begin{gathered}
\mathbb{P}(\omega)^{T} \mathbb{v}(\omega)=\mathbb{v}(\omega), \\
\mathbb{P}(\omega) \mathbb{u}(\omega)=\mathbb{u}(\omega) .
\end{gathered}
$$

Because $\mathbb{P}(\omega)$ is row stochastic for any $\omega \in[0,1]$, $\mathfrak{u}(\omega)=\mathfrak{u}=[1, \ldots, 1]^{T}$ is a right eigenvector with eigenvalue $\lambda_{1}(\omega)=\lambda_{1}=1$. That is, both are independent of $\omega$, and we can drop $\omega$ as an argument. (This will be more rigorously supported in a theorem below). 
Provided that $\mathbb{P}(\omega)$ is a nonnegative irreducible matrix, $\mathbb{V}(\omega)$ and $\mathbb{u}(\omega)$ are uniquely defined and have positive entries [41]. Note that this explicitly assumes $\omega \in(0,1)$. We denote the $\omega \rightarrow 0^{+}$limits of $\mathbb{v}(\omega)$ and $\mathbb{u}(\omega)$ by $\mathbb{u}\left(0^{+}\right)$. However, when $\omega=0$ (i.e., $\omega$ is exactly zero), then $\mathbb{P}(\omega)$ is not irreducible. We provide the following theorem to characterize the eigenspace associated with $\lambda_{1}=1$ in this case.

Theorem B.1. Let $\mathbb{P}(\omega)$ be a supracentrality matrix of a multiplex Markov chain and assume that each intralayer transition matrix $\mathbf{P}^{(i)}$ is nonnegative, irreducible. Then the geometric and algebraic multiplicity of eigenvalue $\lambda_{1}=1$ of $\mathbb{P}(0)$ are both $I$ (recall that $I$ is the number of intralayer Markov chains), and the left and right eigenspaces are spanned by orthogonal eigenvectors

$$
\begin{aligned}
& \mathbb{v}^{(i)}=\mathbf{e}^{(i)} \otimes \mathbf{v}^{(i)}, \\
& \mathfrak{u}^{(i)}=\mathbf{e}^{(i)} \otimes \mathbf{u}^{(i)},
\end{aligned}
$$

respectively, where $\mathbf{e}^{(i)}$ denotes the unit vector (i.e., all entries are zero except for the $i$-th entry, which is a 1) and $\otimes$ denotes the Kronecker product.

Remark 1. We refer to the vectors $\mathbb{v}^{(i)}$ and $u^{(i)}$ as "block vectors," and they consist of zeros, except in the $i$ th blocks, which are $\mathbf{v}^{(i)}$ and $\mathbf{u}^{(i)}$, respectively.

Proof. First, we show that $\mathbb{V}^{(i)}$ and $\mathrm{u}^{(i)}$ are left and right eigenvectors of $\mathbb{P}(0)$ corresponding to the eigenvalue $\lambda_{1}=1$,

$$
\begin{aligned}
\mathbb{P}(0) \mathfrak{u}^{(i)} & =\mathbf{e}^{(i)} \otimes \mathbf{P}^{(i)} \mathbf{u}^{(i)} \\
& =\mathbf{e}^{(i)} \otimes \mu_{1}^{(i)} \mathbf{u}^{(i)} \\
& =\mathfrak{u}^{(i)}
\end{aligned}
$$

and

$$
\begin{aligned}
\mathbb{P}(0)^{T} \mathbb{V}^{(i)} & =\mathbf{e}^{(i)} \otimes\left[\mathbf{P}^{(i)}\right]^{T} \mathbf{v}^{(i)} \\
& =\mathbf{e}^{(i)} \otimes \mu_{1}^{(i)} \mathbf{v}^{(i)} \\
& =\mathbb{V}^{(i)}
\end{aligned}
$$

It is also straightforward to show that these sets of eigenvectors are orthogonal:

$$
\begin{aligned}
{\left[\mathbb{v}^{(i)}\right]^{T} \mathbb{V}^{(j)} } & =\left(\mathbf{e}^{(i)} \otimes \mathbf{v}^{(i)}\right)^{T}\left(\mathbf{e}^{(j)} \otimes \mathbf{v}^{(j)}\right) \\
& =\left(\left[\mathbf{e}^{(i)}\right]^{T} \otimes\left[\mathbf{v}^{(i)}\right]^{T}\right)\left(\mathbf{e}^{(j)} \otimes \mathbf{v}^{(j)}\right) \\
& =\left[\mathbf{e}^{(i)}\right]^{T} \mathbf{e}^{(j)} \otimes\left[\mathbf{v}^{(i)}\right]^{T} \mathbf{v}^{(j)} \\
& =\delta_{i j}
\end{aligned}
$$

and

$$
\begin{aligned}
{\left[\mathfrak{u}^{(i)}\right]^{T} \mathfrak{u}^{(j)} } & =\left(\mathbf{e}^{(i)} \otimes \mathbf{u}^{(i)}\right)^{T}\left(\mathbf{e}^{(j)} \otimes \mathbf{u}^{(j)}\right) \\
& =\left(\left[\mathbf{e}^{(i)}\right]^{T} \otimes\left[\mathbf{u}^{(i)}\right]^{T}\right)\left(\mathbf{e}^{(j)} \otimes \mathbf{u}^{(j)}\right) \\
& =\left[\mathbf{e}^{(i)}\right]^{T} \mathbf{e}^{(j)} \otimes\left[\mathbf{u}^{(i)}\right]^{T} \mathbf{u}^{(j)} \\
& =\delta_{i j} .
\end{aligned}
$$

These results use that the Kronecker-product identity $(a \otimes$ $b)(c \otimes d)=a c \otimes b d$ (assuming the dimensions appropriately match).

Provided that each $\mathbf{P}^{(i)}$ is nonnegative and irreducible, the dominant eigenvalue $\mu_{1}^{(i)}=1$ of each $\mathbf{P}^{(i)}$ has geometric and algebraic multiplicity equal to 1 . Thus the eigenvalue $\lambda_{1}=1$ of $\mathbb{P}(0)$ has geometric and algebraic multiplicity equal to $I$. The sets of eigenvectors $\left\{\mathbb{v}^{(i)}\right\}$ and $\left\{u^{(i)}\right\}$ are eigenbases for the left and right eigenspaces for $\lambda_{1}=1$.

Next, we present our main analytical result for when there is a separation of time scales and transitions are far more likely to utilize an intralayer Markov chain versus an interlayer one.

Theorem B.2. Let $\mathbb{P}(\omega)$ be a supracentrality matrix of a multiplex Markov chain and assume each intralayer transition matrix $\mathbf{P}^{(i)}$ is nonnegative, irreducible, and has a dominant eigenvalue such that $\mu_{1}^{(i)}=1$. We define $\mathbb{v}\left(0^{+}\right)=$ $\lim _{\omega \rightarrow 0^{+}} \mathbb{V}(\omega)$ as the limiting left eigenvector of $\mathbb{P}(\omega)$. Then

$$
\mathbb{V}\left(0^{+}\right)=\sum_{i=1}^{I} \tilde{\alpha}_{i} \mathbb{V}^{(i)},
$$

where the vector $\tilde{\boldsymbol{\alpha}}=\left[\alpha_{1}, \ldots, \alpha_{I}\right]^{T}$ has positive entries that satisfy $\sum_{i=1}^{I} \tilde{\alpha}_{i}$ and is a unique solution to

$$
\tilde{\boldsymbol{\alpha}}^{T} \tilde{\boldsymbol{X}}=\tilde{\boldsymbol{\alpha}}^{T}
$$

with $\tilde{X}_{i j}=\sum_{n=1}^{N} \tilde{P}_{i j}^{(n)} v_{n}^{(i)}$.

Remark 2. Since each $\tilde{\boldsymbol{P}}^{(n)}$ is row stochastic, matrix $\tilde{\boldsymbol{X}}$ is also row stochastic:

$$
\begin{aligned}
\sum_{j} \tilde{X}_{i j} & =\sum_{n=1}^{N} \sum_{j} \tilde{P}_{i j}^{(n)} v_{n}^{(i)} \\
& =\sum_{n=1}^{N} v_{n}^{(i)} \\
& =1 .
\end{aligned}
$$

It follows that it $[1, \ldots, 1]^{T}$ is a right eigenvector of $\tilde{\boldsymbol{X}}$ for an eigenvalue equal to 1 . Therefore $\tilde{\boldsymbol{X}}$ is an "effective" interlayer transition matrix that represents a type of weighted aggregation of the interlayer Markov chains $\left\{\tilde{\boldsymbol{P}}^{(n)}\right\}$.

Remark 3. When the intralayer Markov chains are uniformly coupled, i.e., $\tilde{\boldsymbol{P}}^{(n)}=\tilde{\boldsymbol{P}}$ for each node $n$, it then follows that $\tilde{\boldsymbol{X}}=\tilde{\boldsymbol{P}}$ and $\tilde{\boldsymbol{\alpha}}=\tilde{\boldsymbol{v}}$, which is the left dominant eigenvector of $\tilde{\boldsymbol{P}}$.

Remark 4. When the intralayer Markov chains have doubly stochastic transition matrices, i.e., $\sum_{n} P_{n m}^{(i)}=\sum_{m} P_{n m}^{(i)}=1$ for each node $i$, then $v_{n}^{(i)}=1 / N$ for each $i$ and $\tilde{\boldsymbol{X}}$ is the mean intralayer transition matrix.

Proof. Theorem B. 1 proved that $\lambda_{1}$ has a $P$-dimensional left dominant eigenspace that are spanned by the left eigenvectors $\mathbb{V}^{(i)}$. The continuity of eigenvector spaces [43] ensures that $\mathbb{V}\left(0^{+}\right)$converges to lie within this subspace, which implies Eq. (B7). We now prove that the constants $\tilde{\alpha}_{i}$ satisfy Eq. (B8).

We Taylor expand $\mathbb{v}(\omega)$ for small $\omega$ as

$$
\mathbb{v}(\omega)=\sum_{k=0}^{K} \omega^{k} \mathbb{v}_{k}+\mathcal{O}\left(\omega^{K+1}\right) .
$$

Successive terms in this expansion represent higher-order derivatives of $\mathbb{v}(\omega)$ with respect to $\omega$, and we assume that $\mathbb{V}(\omega)$ has the appropriate smoothness [i.e., $\mathbb{V}(\omega) \in C^{(k)}(0,1)$ ]. The $\omega \rightarrow 0^{+}$limit of $\mathbb{v}(\omega)$ then becomes $\mathbb{v}_{0}=\mathbb{v}\left(0^{+}\right)$. Focusing on the first-order approximation, we insert $\mathbb{v}(\omega) \approx$ 
$\mathbb{v}_{0}+\omega \mathbb{v}_{1}$ into the eigenvalue equation

$$
\mathbb{V}(\omega)=\mathbb{P}(\omega)^{T} \mathbb{v}(\omega)
$$

to obtain

$$
\begin{aligned}
\mathbb{v}_{0}+\omega \mathbb{V}_{1}= & (1-\omega) \mathbb{P}(0)^{T}\left[\mathbb{v}_{0}+\omega \mathbb{V}_{1}\right] \\
& +\omega \sum_{n}\left[\tilde{\boldsymbol{P}}^{(n)} \otimes \mathbf{E}^{(n)}\right]^{T}\left[\mathbb{v}_{0}+\omega \mathbb{V}_{1}\right] \\
= & \mathbb{P}(0)^{T} \mathbb{V}_{0}+\omega \mathbb{P}(0)^{T} \mathbb{V}_{1}-\omega \mathbb{P}(0)^{T} \mathbb{v}_{0} \\
& -\omega^{2} \mathbb{P}(0)^{T} \mathbb{V}_{1}+\omega \sum_{n}\left[\tilde{\boldsymbol{P}}^{(n)} \otimes \mathbf{E}^{(n)}\right]^{T} \mathbb{V}_{0} \\
& +\omega^{2} \sum_{n}\left[\tilde{\boldsymbol{P}}^{(n)} \otimes \mathbf{E}^{(n)}\right]^{T} \mathbb{V}_{1}
\end{aligned}
$$

The second-order terms will be negligible as $\omega \rightarrow 0$, and so we separately collect the zeroth-order and first-order terms in $\omega$ to obtain two consistency equations:

$$
\mathbb{v}_{0}=\mathbb{P}(0)^{T} \mathbb{v}_{0}
$$

and

$$
\mathbb{v}_{1}=\mathbb{P}(0)^{T} \mathbb{v}_{1}-\mathbb{P}(0)^{T} \mathbb{v}_{0}+\sum_{n}\left[\tilde{\boldsymbol{P}}^{(n)} \otimes \mathbf{E}^{(n)}\right]^{T} \mathbb{v}_{0}
$$

The consistency equation arising for the zeroth-order terms is exactly the eigenvalue equation with $\omega=0$, as expected. It implies a solution of the form given by Eq. (B7).
To proceed, we left multiply the consistency equation arising from the first-order terms by $\mathrm{u}^{(i)}$, yielding

$$
\begin{aligned}
{\left[\mathfrak{u}^{(i)}\right]^{T} \mathbb{v}_{1}=} & {\left[\mathfrak{u}^{(i)}\right]^{T} \mathbb{P}(0)^{T} \mathbb{v}_{1}-\left[\mathfrak{u}^{(i)}\right]^{T} \mathbb{P}(0)^{T} \mathbb{v}_{0} } \\
& +\sum_{n}\left[\mathfrak{u}^{(i)}\right]^{T}\left(\tilde{\boldsymbol{P}}^{(n)} \otimes \mathbf{E}^{(n)}\right)^{T} \mathbb{v}_{0} .
\end{aligned}
$$

However, $\left[\mathfrak{u}^{(i)}\right]^{T} \mathbb{P}(0)^{T}=\left[\mathrm{u}^{(i)}\right]^{T}$ and the term on the lefthand side is canceled by the first term on the right-hand side, which yields

$$
\left[\mathfrak{u}^{(i)}\right]^{T} \mathbb{v}_{0}=\sum_{n} \mathfrak{u}^{(i)}\left(\tilde{\boldsymbol{P}}^{(n)} \otimes \mathbf{E}^{(n)}\right)^{T} \mathbb{v}_{0} .
$$

To simplify the left-hand side of Eq. (B16), we use

$$
\begin{aligned}
{\left[\mathrm{u}^{(i)}\right]^{T} } & =\left[\mathbf{e}^{(i)} \otimes \mathbf{u}^{(i)}\right]^{T} \\
& =\left[\mathbf{e}^{(i)}\right]^{T} \otimes\left[\mathbf{u}^{(i)}\right]^{T}
\end{aligned}
$$

and $\mathbb{v}_{0}=\sum_{j} \tilde{\alpha}_{j}\left(\mathbf{e}^{(j)} \otimes \mathbf{v}^{(j)}\right)$ to obtain

$$
\begin{aligned}
{\left[\mathrm{u}^{(i)}\right]^{T} \mathbb{v}_{0} } & =\sum_{j} \tilde{\alpha}_{j}\left(\left[\mathbf{e}^{(i)}\right]^{T} \otimes\left[\mathbf{u}^{(i)}\right]^{T}\right)\left(\mathbf{e}^{(i)} \otimes \mathbf{v}^{(j)}\right) \\
& =\sum_{j} \tilde{\alpha}_{j}\left(\left[\mathbf{e}^{(i)}\right]^{T} \mathbf{e}^{(j)}\right) \otimes\left(\left[\mathbf{u}^{(i)}\right]^{T} \mathbf{v}^{(j)}\right) \\
& =\sum_{j} \tilde{\alpha}_{j} \delta_{i j}\left(\left[\mathbf{u}^{(i)}\right]^{T} \mathbf{v}^{(j)}\right) \\
& =\tilde{\alpha}_{i} .
\end{aligned}
$$

[Recall that $u_{n}^{(i)}=1$ for each $n$ and $\sum_{n} v_{n}^{(i)}=1$ ]. Finally, we obtain $\tilde{\boldsymbol{\alpha}}^{T} \tilde{\boldsymbol{X}}=\tilde{\boldsymbol{\alpha}}^{T}$ by setting Eq. (B18) equal to the following

simplification for the right-hand side of Eq. (B16),

$$
\begin{aligned}
\sum_{n}\left[\mathrm{u}^{(i)}\right]^{T}\left(\left[\tilde{\boldsymbol{P}}^{(n)}\right]^{T} \otimes\left[\mathbf{E}^{(n)}\right]^{T}\right) \mathbb{v}_{0} & =\sum_{n}\left[\mathrm{u}^{(i)}\right]^{T}\left(\left[\tilde{\boldsymbol{P}}^{(n)}\right]^{T} \otimes\left[\mathbf{E}^{(n)}\right]^{T}\right) \sum_{j} \tilde{\alpha}_{j} \mathbb{V}^{(j)} \\
& =\sum_{j} \tilde{\alpha}_{j} \sum_{n}\left(\left[\mathbf{e}^{(i)}\right]^{T} \otimes\left[\mathbf{u}^{(i)}\right]^{T}\right)\left(\left[\tilde{\boldsymbol{P}}^{(n)}\right]^{T} \otimes\left[\mathbf{E}^{(n)}\right]^{T}\right)\left(\mathbf{e}^{(j)} \otimes \mathbf{v}^{(j)}\right) \\
& =\sum_{j} \tilde{\alpha}_{j} \sum_{n}\left(\left[\mathbf{e}^{(i)}\right]^{T}\left[\tilde{\boldsymbol{P}}^{(n)}\right]^{T} \otimes\left[\mathbf{u}^{(i)}\right]^{T}\left[\mathbf{E}^{(n)}\right]^{T}\right)\left(\mathbf{e}^{(j)} \otimes \mathbf{v}^{(j)}\right) \\
& =\sum_{j} \tilde{\alpha}_{j} \sum_{n}\left(\left[\mathbf{e}^{(i)}\right]^{T}\left[\tilde{\boldsymbol{P}}^{(n)}\right]^{T} \mathbf{e}^{(j)}\right) \otimes\left(\left[\mathbf{u}^{(i)}\right]^{T}\left[\mathbf{E}^{(n)}\right]^{T} \mathbf{v}^{(j)}\right) \\
& =\sum_{j} \tilde{\alpha}_{j} \sum_{n}\left[\tilde{P}^{(n)}\right]_{i j}^{T} u_{n}^{(i)} v_{n}^{(j)} \\
& =\sum_{j} \tilde{\alpha}_{j} \sum_{n} \tilde{P}_{j i}^{(n)} v_{n}^{(j)} \\
& =\sum_{j} \tilde{\alpha}_{j} X_{j i} .
\end{aligned}
$$



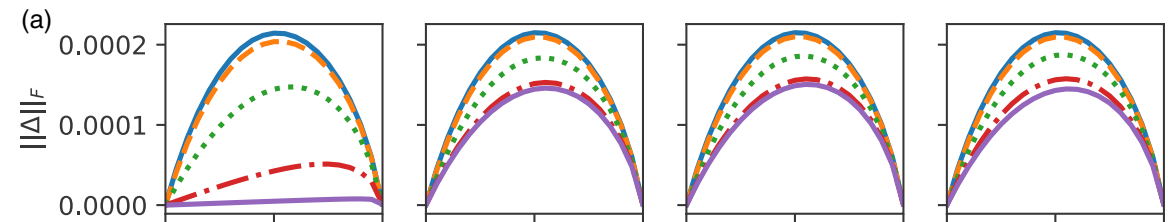

(b)

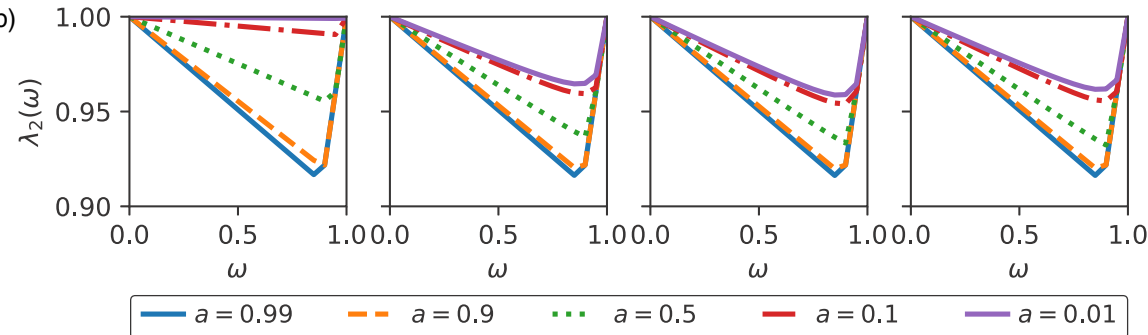

FIG. 8. Optimality of multiplex imbalance and convergence rate for MMC model of a functional brain network (a) Total multiplex imbalance $\|\Delta\|_{F}$ and (b) convergence rate $\lambda_{2}(\omega)$ vs $\omega$ for the same intralayer Markov chains as in Figs. 5-7, except with node-specific interlayer Markov chains with transition matrices having entries: $\tilde{P}_{i j}^{(n)}=$ $\left(1-a_{n}\right)$ if $i=j, \tilde{P}_{i j}^{(n)}=a_{n}$ if $i=|j \pm 1|$, and $\tilde{P}_{i j}^{(n)}=0$ otherwise. Each column depicts results for a different strategy for choosing the $a_{n}$ values, and each depends on a parameter $a \in[0,1]$ : (left) identical $a_{n}$; (center-left) increasing $a_{n}$; (center-right) decreasing $a_{n}$; and (right) random $a_{n}$.

\section{APPENDIX C: EXTENDED STUDY OF MMC MODEL FOR FREQUENCY-MULTIPLEXED FUNCTIONAL BRAIN NETWORKS}

Here, we provide further results and insights on the optimality of multiplex imbalance for MMC models of frequencymultiplexed functional brain networks using empirical data from [13]. Unlike our study in Sec. V, we now couple each node across layers using node-specific interlayer Markov chains with transition matrices $\tilde{\boldsymbol{P}}^{(n)}$ having entries

$$
\tilde{P}_{i j}^{(n)}=\left\{\begin{aligned}
1-a_{n}, & i=j \\
a_{n}, & |i-j|=1 \text { and } i \in\{1, N\} \\
a_{n} / 2, & |i-j|=1 \text { and } i \notin\{1, N\} \\
0, & \text { otherwise }
\end{aligned}\right.
$$

That is, we couple the Markov chain layer for each frequency band with those of the adjacent frequency bands with weight $a_{n} / 2$ (with the exception of the first and last layers, which are coupled by $a_{n}$ ). We choose the $a_{n}$ values in the same way as we described in Sec. IV. Because we would like to introduce correlations between the $a_{n}$ values and the nodes' intralayer degrees $d_{n}^{(i)}$, we permuted the nodes indices so that their mean intralayer degrees, $I^{-1} \sum_{i} d_{n}^{(i)}$, decrease monotonically with $n=1,2 \ldots, N$.

In Fig. 8, we show a plot that is analogous to Fig. 3, except we now show results for the brain dataset. The top panels depict the total multiplex imbalance $\|\Delta\|_{F}$, while the lower panels depict convergence rate $\lambda_{2}(\omega)$, both as a function of $\omega$. Each column in Fig. 8 depicts curves for a different strategy for choosing the $a_{n}$ values, and each depends on a parameter $a \in[0,1]$ (see the description of Fig. 3 in the main to remind yourself of these four strategies). Observe that in this case, the locations $\omega_{\Delta}^{*}$ and $\omega_{\lambda_{2}}^{*}$ of optima occur at very different values of $\omega$, highlighting that optimality can more complicated when the number of Markov-chain layers increases (in this case there are $I=7$ layers, whereas Fig. 3 in the main text shows results for $I=2$ layers).

To gain insight into how optimality may depend on the number of layers, next we next study how the net flow $\Delta_{p q}$ across each edge obtains its own optimum at some value $\omega$. In Fig. 9, we plot each $\Delta_{p q}$ versus $\omega$, and we separately plot the values for (a) intralayer edges [i.e., edges between node-layer pairs $\left(n_{p}, i_{p}\right)$ and $\left(n_{q}, i_{q}\right)$ with $\left.i_{q}=i_{p}\right]$ and (b) interlayer edges [i.e., edges between node-layer pairs $\left(n_{p}, i_{p}\right)$ and $\left(n_{q}, i_{q}\right)$ with $n_{q}=n_{p}$ ]. That is, we create the decomposition $\Delta=\Delta^{\text {(intra) }}+$ $\Delta^{\text {(inter) }}$. Because the values $\Delta_{p q}$ come in pairs having opposite signs, i.e., since $\Delta_{p q}=-\Delta_{q p}$, we only plot positive $\Delta_{p q}$. Interesting, we find for all edges that the sign of $\Delta_{p q}$ does not change. That is, the directions of net flows do not switch as $\omega$ varies, although it remains unclear if this is a general property of all multiplex networks.

Observe in Figs. 9(a) and 9(b) that the $\Delta_{p q}$ value for some edges become much larger than those of others, and that the largest values obtain their maximum near $\omega \approx 0.5$. In Fig. 9(c), we plot $\left\|\Delta^{\text {(intra) }}\right\|_{F}$ and $\left\|\Delta^{\text {(inter) }}\right\|_{F}$, where $\Delta_{p q}^{(\text {intra })}=\Delta_{p q}$ if the edge between $p$ and $q$ is an intralayer edge and $\Delta_{p q}^{\text {(intra) }}=0$ otherwise. $\Delta_{p q}^{\text {(inter) }}$ is similarly defined. Note that $\|\Delta\|_{F}^{2}=\left\|\Delta^{\text {(intra) }}\right\|_{F}^{2}+\left\|\Delta^{\text {(inter) }}\right\|_{F}^{2}$. Observe that the $\Delta_{p q}^{\text {(intra) }}$ values are on average about one-tenth as large as the $\Delta_{p q}^{\text {(inter) }}$ values.
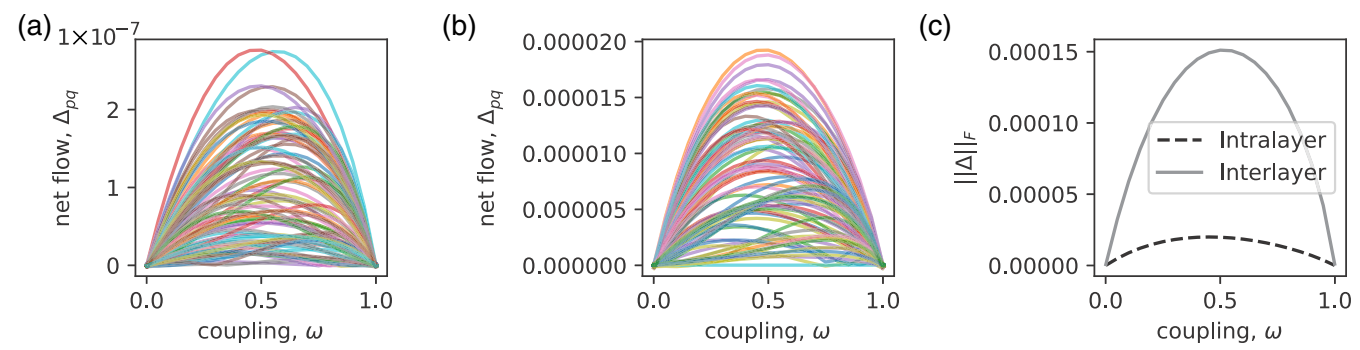

FIG. 9. Optimality of $\Delta_{p q}$ for each edge in the MMC representing the brain data. We plot the positive $\Delta_{p q}$ values versus $\omega$ for each (a) intralayer and (b) interlayer edge. In (c), we separate $\Delta=\Delta^{\text {(intra) }}+\Delta^{\text {(inter) }}$ into two matrices, one corresponding to intralayer edges and the other corresponding to interlayer edges, and we plot their respective Frobenius norms vs $\omega$. 

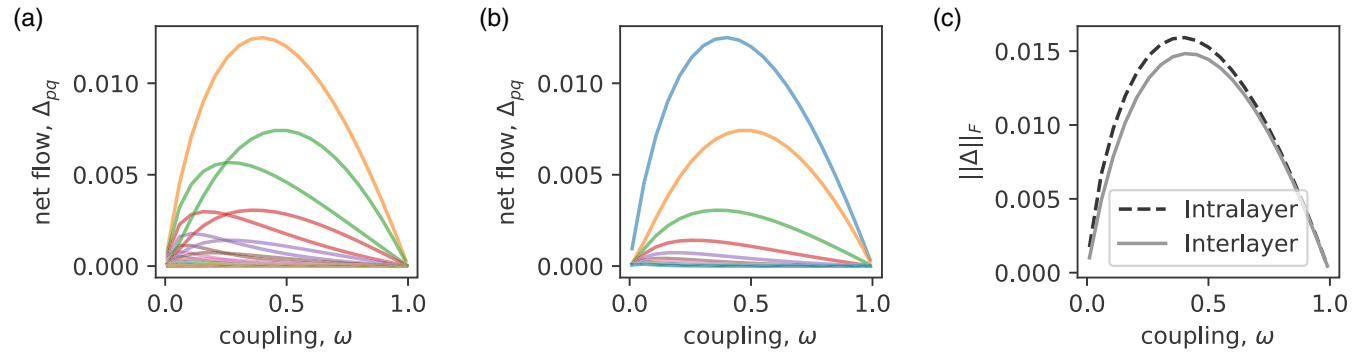

FIG. 10. Optimality of $\Delta_{p q}$ for each edge for the MMC shown in Fig. 1. The results are similar to those in Fig. 9.

In Fig. 10, we show plots that are analogous to Fig. 9, except they are for the MMC from Fig. 1. Observe in Figs. 10(a) and $10(\mathrm{~b})$ that the $\Delta_{p q}$ value for some edges become much larger than those of others, and that the largest values obtain their maximum near $\omega \approx 0.45$. In contrast, the $\Delta_{p q}$ values that never become large tend to obtain their maximums at smaller $\omega \ll 0.45$. Observe in Fig. 10 (c) that the $\Delta_{p q}$ values for interand intralayer edges for this two-layer MMC are about the same magnitude. This contrasts Fig. 9(c) where the $\Delta_{p q}$ values are much larger for the interlayer edges.
[1] G. Falkovich, Fluid Mechanics: A Short Course for Physicists (Cambridge University Press, New York, 2011).

[2] E. Cozzo, G. F. De Arruda, F. A. Rodrigues, and Y. Moreno, Multiplex Networks: Basic Formalism and Structural Properties (Springer, Berlin, 2018).

[3] S. Boccaletti, G. Bianconi, R. Criado, C. Del Genio, J. GómezGardeñes, M. Romance, I. Sendina-Nadal, Z. Wang, and M. Zanin, Phys. Rep. 544, 1 (2014).

[4] M. Kivelä, A. Arenas, M. Barthelemy, J. P. Gleeson, Y. Moreno, and M. A. Porter, J. Complex Networks 2, 203 (2014).

[5] D. Taylor, F. Klimm, H. A. Harrington, M. Kramár, K. Mischaikow, M. A. Porter, and P. J. Mucha, Nat. Commun. 6, 7723 (2015)

[6] E. Strano, S. Shai, S. Dobson, and M. Barthelemy, J. R. Soc., Interface 12, 20150651 (2015).

[7] A. Solé-Ribalta, A. Arenas, and S. Gómez, New J. Phys. 21, 035003 (2019).

[8] Y. Y. Haimes and P. Jiang, J. Infrast. Syst. 7, 1 (2001).

[9] D. Krackhardt, Social Networks 9, 109 (1987).

[10] M. De Domenico, V. Nicosia, A. Arenas, and V. Latora, Nat. Commun. 6, 6864 (2015).

[11] D. Taylor, S. Shai, N. Stanley, and P. J. Mucha, Phys. Rev. Lett. 116, 228301 (2016).

[12] D. Taylor, R. S. Caceres, and P. J. Mucha, Phys. Rev. X 7, 031056 (2017).

[13] J. Guillon, Y. Attal, O. Colliot, V. La Corte, B. Dubois, D. Schwartz, M. Chavez, and F. D. V. Fallani, Sci. Rep. 7, 10879 (2017).

[14] D. Soriano-Paños, L. Lotero, A. Arenas, and J. GómezGardeñes, Phys. Rev. X 8, 031039 (2018).

[15] J. G. Kemeny and J. L. Snell, Markov Chains (Springer-Verlag, New York, 1976).

[16] D. G. Kendall, Ann. Math. Stat. 24, 338 (1953).

[17] J. Kingman, J. Appl. Prob. 6, 1 (1969).

[18] W. R. Gilks, S. Richardson, and D. Spiegelhalter, Markov Chain Monte Carlo in Practice (Chapman and Hall/CRC, London, 1995).

[19] L. Tierney, Ann. Stat. 22, 1701 (1994).
[20] R. Parr and S. J. Russell, in Advances in Neural Information Processing Systems (ACM, 1998), pp. 1043-1049.

[21] J.-C. Delvenne, S. N. Yaliraki, and M. Barahona, Proc. Natl. Acad. Sci. U.S.A. 107, 12755 (2010).

[22] M. T. Schaub, J.-C. Delvenne, S. N. Yaliraki, and M. Barahona, PloS One 7, e32210 (2012).

[23] P. J. Mucha, T. Richardson, K. Macon, M. A. Porter, and J.-P. Onnela, Science 328, 876 (2010).

[24] S. Gómez, A. Díaz-Guilera, J. Gómez-Gardeñes, C. J. PérezVicente, Y. Moreno, and A. Arenas, Phys. Rev. Lett. 110, 028701 (2013).

[25] A. Solé-Ribalta, M. De Domenico, N. E. Kouvaris, A. DiazGuilera, S. Gomez, and A. Arenas, Phys. Rev. E 88, 032807 (2013).

[26] F. Radicchi and A. Arenas, Nat. Phys. 9, 717 (2013).

[27] M. De Domenico, A. Solé-Ribalta, S. Gómez, and A. Arenas, Proc. Natl. Acad. Sci. U.S.A. 111, 8351 (2014).

[28] A. Tejedor, A. Longjas, E. Foufoula-Georgiou, T. T. Georgiou, and Y. Moreno, Phys. Rev. X 8, 031071 (2018).

[29] G. Cencetti and F. Battiston, New J. Phys. 21, 035006 (2019).

[30] I. Trpevski, A. Stanoev, A. Koseska, and L. Kocarev, New J. Phys. 16, 113063 (2014).

[31] M. De Domenico, A. Solé-Ribalta, E. Omodei, S. Gómez, and A. Arenas, Nat. Commun. 6, 6868 (2015).

[32] A. Solé-Ribalta, M. De Domenico, S. Gómez, and A. Arenas, Physica D 323, 73 (2016).

[33] D. Taylor, S. A. Myers, A. Clauset, M. A. Porter, and P. J. Mucha, Multiscale Model. Simul. 15, 537 (2017).

[34] D. R. DeFord and S. D. Pauls, J. Complex Networks 6, 353 (2018).

[35] D. Taylor, M. A. Porter, and P. J. Mucha, Supracentrality analysis of temporal networks with directed interlayer coupling, in Temporal Network Theory, edited by P. Holme and J. Saramäki (Springer International, Cham, Switzerland, 2019), pp. 325344.

[36] D. Taylor, M. A. Porter, and P. J. Mucha, arXiv:1904.02059 (2019).

[37] M. De Domenico, C. Granell, M. A. Porter, and A. Arenas, Nat. Phys. 12, 901 (2016). 
[38] D. Taylor, https://github.com/taylordr/multiplexMarkovChains. [39] P. S. Skardal, D. Taylor, and J. Sun, Phys. Rev. Lett. 113, 144101 (2014).

[40] D. Taylor, P. S. Skardal, and J. Sun, SIAM J. Appl. Math. 76, 1984 (2016).

[41] R. B. Bapat and T. E. S. Raghavan, Nonnegative Matrices and Applications, Encyclopedia of Mathematics and its Applica- tions Vol. 64 (Cambridge University Press, Cambridge, UK, 1997).

[42] P. Sahoo and T. Riedel, Mean Value Theorems and Functional Equations (World Scientific, Singapore, 1998).

[43] T. Kato, Perturbation Theory for Linear Operators, Classics in Mathematics Vol. 132 (Springer Science \& Business Media, New York, 2013). 